\title{
Die Verlagerung der Pupille durch Iridodesis
}

\section{von}

Hofrath Dr. Pagenstecher zu Wiesbaden.

Die nachfolgenden Zeilen bezwecken, meine Erfahrungen mitzutheilen, die ich im Laufe der letzten Jahre über die Erfolge der Verlagerungen der Pupille durch Iridodesis bei eivigen krankhaften Veränderungen der Cornea und und des Linsensystems gewonnen habe.

Schon im VI. Bd. II. Abth. des Archiv's wurde von meinem früheren Assistenzarzte Dr. Berlin eine Arbeit hierüber veröffentlicht und wenn ich bisher zögerte meine Ansichten über dies neue Verfahren auszusprechen, so geschah es nur deshalb; weil ich erst eine grössere Anzahl von Fällen der Controlle unterwerfen wollte, als bei der ersten Mittheilung Berlin's zu Gebote standen.

Die Idee dazu wurde bei mir angeregt durch die Mittheilungen Critchet's' in den London ophthalmic hospital reports, nach welcher derselbe bei partiellen Hornhauttrübungen zum Zwecke der Verlagerung der Pupille gegenüber einer gesunden Hornhautparthie eine Iridoencleisis vornimmt und sich zum Behufe der Fixirung der Iris in der Hornhautwunde einer Fadenschlinge bedient, die er um den prolabirten Theil der Iris anlegt. Durch diese Unterbindung der Iris ausserhalb der Cor- 
nealwunde wurde das prolabirte Stück mortificirt und gleichzeitig die Iris in die Corneawunde eingetheilt. Critshet stellt die Indication zu diesem Verfahren bei allen den Fällen von partiellen Cornealtrübungen, wobei nach erhaltener, theilweiser oder vollständiger Beweglichkeit des Pupillarrandes die Pupille selbst von der leucomatösen Narbe bedeckt wird; also solche Fälle, wo man bisher eine künstliche Pupille durch Iridectomie anlegte.

Der durch das neue Verfahren erzielte Vortheil besteht darin, dass man eine Pupille erhält, deren Rand theilweise oder vollständig durch den Sphincter gebildet wird und somit dem einfallenden Lichte durch eine Contraction entsprechen kann, während eine durch Iridectomie angelegte Pupille nicht nur einen Theil oder das ganze Quantum Sphincterfasern vernichtet, sondern auch noch Begrenzungen erbält, die die Verkleinerung der Pupille bei Lichteinfall unmöglich machen. Es ist hinlänglich bekannt, wie sehr diffuses Licht, mag dasselbe von Corneal- oder Linsentrübungen ausgehen, die genaue Perception hindert, selbst lichte Cornealtrübungen erzeugen oft grosse Blendungs-Erscheinungen und verursachen durch Diffusion der Lichtstrahlen ein bedeutendes Hinderniss für das deutliche Sehen; namentlich macht sich dies beim Sehen im Freien, bei Sonnenlicht, bei reflectirtem Licht u. s. f. denen, die daran leiden, auf eine empfindliche Weise bemerklich. Die sofort eintretende Lichtscheu stellt solche Personen in gleiche Linie mit an hochgradiger Amblyopie Leidenden, während sie bei geringerer Lichtintersität oft noch ein überraschend gutes Sehvermögen haben.

Durch Bildung einer künstlichen Pupille mittelst Iridectomie (ohne gleichzeitige Einklemmung der Iris) können wir, selbst wenn wir dieselbe möglichst klein anlegen, diesem Uebelstande nicht vollständig begegnen; 
die Blendungs-Frscheinungen werden nicht vermindert, oft sogar noch vermehrt, da doch der früher vorhandene Pupillarraum, der das von der getrübten Cornea erzeugte diffuse Licht zur Retina führte, nicht verkleinert, sondern sehr häufig die das diffuse Licht bewirkende Fläche noch vergrössert wird. Ausserdem und worauf es hier vorzüglich ankommt, entbehrt eine durch Iridectomie angelegte Pupille in hohem Grade des Beschützers vor diffusem Lichte, des Sphincter pupillae. Eine solche Pupille zieht sich nur unvollkommen bei Lichteinfall zusammen und schliesst niemals, selbst wenn sie noch so klein angelegt wird, die Blendung ganz aus.

Critchet sucht diesen Beschützer vor diffusem Lichte möglichst"nutzbar zu machen, indem er ihn erhält und ihm gerade eine Stelle anweisst, wo er seine Thätigkeit bei einfallendem Lichtc, wie bei einer normalen Pupille entfaltet. Während Critchet nur bei Hornhauttrübungen, wobei er die Eröffnung der vorderen Kammer vor der cornea selbst aus vornimmt, dieses Verfahren verwendet, kann ich dasselbe auch bei gewissen stationären Linsentrübungen mit einer abweichenden Modification empfehlen.

Hauptsächlich ist es der Schichtstaar, Catararta zonularis, vor Arlt stationärer Kernstaar jugendlicher Individuen genannt, wo die Iridodesis ihren Platz findet. früher suchte man diese Staarformen wie die gewöhnlichen Linsenstaare durch Extraction oder Dissision zu entfernen. v. Graefe wandte zuerst die Iridectomie dabei an, indem er eine Randpupille anlegte. Wenn auch die durch Iridectomie erzeugte Pupille um ein Beträchtliches das Sehvermögen namentlich in die Nähe verbesserte, so wurden doch dadurch die Blendungs-Erscheinungen beim Sehen in die Entfernung und starker Beleuchtung nicht beseitigt. Man befand sich also einem Schichtstaarkranken gegenüber in einem Dilemma. Entfernte man die 
Linse durch eine Staaroperation, so war der Kranke, abgesehen von der durch ihre Gefährlichkeit immerhin in's Gewicht fallenden Staaroperation genöthigt zeitlebens zum Behufe des deutlichen Sehens sich einer Staarbrille zu bedienen. Machte man eine Iridectomie, so wurde zwar das Sehen in der Nähe verbessert, allein wegen der gelegentlich eintretenden starken Blendungs-Erscheinungen blieb doch der Erfolg für den Kranken ein unvollkommener. Beide Unzuträglichkeiten schliesse ich aus durch Verlagerung der Pupille durch Iridodesis.

Durch eine Modification des Critchet'schen Verfahrens gelangt man auf eine sehr einfache Weise dahin,

1) nicht nur die Thätigkeit des Sphincter pupillae zu erhalten, sondern auch

2) den früheren centralen Pupillarraum zu beseitigen und an jeder beliebigen Stelle der Peripherie eine neue Pupille zu schaffen.

Der Modus der Operation besteht darin, dass ich von der Sclera aus mit einer breiten über die Fläche gebogenen Paracentensenadel einen Einstich in die vordere Kammer mache und hierauf einen Prolapsus iridis, wenn sich derselbe beim Zurückziehen des Instrumentes nicht von selbst einstellt, bewirke. Die prolabirte Irisparthie wird dann mit einer Fadenschlinge umgeben und letztere in dem Augenblicke zugezogen, wenn der mit der Pincette gefasste Prolapsus in dem Grade zugezogen ist, dass dadurch der ganze Pupillarraum ausserhalb des Bereichs der Trübung, mag dieselbe von der Cornea oder der Linse ausgehen, verrückt ist.

Es kommt hierbei in hohem Grade die ausgezeichnete Dehnbarkeit der Iris zu statten, denn durch einen Zug mit der Pincette an dem nach innen gelegenen Pupillarrande ist man im Stande, den ganzen Bereich der Pupille fast um das Doppelte ihrer Breite zu verziehen, so dass der äussere Pupillarrand noch dieseits der früheren Stelle 
des inneren Pupillarrandes zu liegen kommt. Man muss dabei die Vorsicht gebrauchen, dass man die Iris möglichst nahe am Pupillarrande anfasst, alsdann folgt der gegenüber liegende Rand mit dem Gewebe der Iris, deren Radialfasern allerdings sehr verlängert werden, nach und man wird auf diese Weise niemals eine Dialyse der Iris von ihrer Ciliarinsertion erhalten. Der um die prolabirte Irisparthie angelegte Faden bleibt liegen und wird durch Necrose der Iris, die sich in der Regel nach 24 bis 36 Stunden einstellt, abgestossen.

Bei der Anlage der Schlinge muss man natürlicher Weise darauf bedacht sein, sie unmittelbar auf dem Bulbus liegend anzuziehen, damit nicht noch eine ununterbundene Parthie der Iris zwischen dem Knoten und der einklemmenden Scleralwunde liegen bleibt; dies verzögert etwas die Vernarbung und giebt leicht zu einer cystenartigen Vortreibung des Irisgewebes von der vorderen Kammer aus Veranlassung. Man umgeht dies am leichtesten, wenn man zum Zuschnüren des Knotens den von Dr. Sch uff t construirten Schlingenträger benutzt. Dieses Instrument erleichtert in hohem Grade die Ausführung der Iridodesis und dürfte einem Jeden, der diese Operation vornehmen will, dringend empfohlen sein.

Im Falle man das längere Verweilen der Ligatur in der Nähe der Cornea oder im Conjunctinalsacke fürchtet, kann man dieselbe schon nach 24 Stunden mit der Scheere coupiren. Die Einklemmung der Iris ist alsdann schon mit hinlänglicher Festigkeit bewerkstelligt. Entzündliche leizungen, wie man sie vermuthen könnte, kommen nach meinen Beobachtungen nicht vor; ich habe jetzt im Ganzen 48mal diese Operation gemacht und noch niemals habe ich eine längere Heilungsperiode beobachtet, als nach einer gewöhnlichen Iridectomie.

Ich erziele durch die Iridodesis eine Pupille, die in physiologischer Beziehung ganz einer normalen entspricht. 
Sie stellt also eigentlich nur eine Verlagerung der normalen Pupille dar, wobei es von dem Willen des Operateurs abhängt, sie an eine Stelle zu verlegen, die es gestattet, dass nur normal gebrochenes Licht der Retina zugeführt wird, mit Vermeidung aller Diffusion. Durch die Möglichkeit, sie so klein wie erforderlich anzulegen, erhält sie zugleich das Hauptpestulat einer künstlichen Pupille, die nicht zugleich als Antiphlogisticum dienen soll. Die Lage derselben in unmittelbarer Nähe der Cornealgrenze und gegenüber einer ziemlich peripherischen Linsenschichte könnte den Einwurf aufkommen lassen, dass das durch diese peripherischen Theile gebrochene Licht ein unvollkommenes Bild auf der Retina erzeugen müsse. Die Beobachtungen aber sprechen dagegen. Die von mir operirten Kranken, die sich einer normalen Energie der Retina erfreuten, sahen sehr deutlich und diejenigen, die Lesen gelernt hatten, konnten die feinsten Nummern der Jäger'schen Schriftscalen lesen. - Auch haben neuere Untersuchungen von Dr. $\mathrm{Kn}$ app in Heidelberg dargethan, dass sowohl die dem Rande zunächst gelegenen Theile der Linse, als der Cornea das Zustandekommen eines klaren Bildes auf der Retina vermitteln können.

Veränderungen in der der Einklemmungsstelle der Iris zunächst gelegenen Cornealparthie in Bezug auf ihre Wölbung, wodurch ihre dioptrische Wirkung pervertirt werden könnte, habe ich nicht beobachtet, da ich immer bemüht war die Wunde im Bereiche der Sclera, mindestens $1 \frac{1}{2}$ bis $2 \mathrm{Mm}$. von der Cornealgrenze anzulegen.

Um die Resultate der Pupillenverlagerung in Bezug auf das Sehvermögen etwes anschaulicher zu machen, will ich einige Krankengeschichten anführen:

1. Anna R., 36 Jahre, seit ihrer Geburt schwachsichtig, bisher als arm und arbeitsunfähig durch den öffentlichen Armenfond unterhalten, kann weder lesen 
noch schreiben, da sie wegen zu grosser Gesichtsschwäche vom Besuche der Schule dispensirt worden war, übrigens gesund, stark und kräftig gebaut, leidet beiderseits an Schichtstaar; die ziemlich saturirte Trübung des Kerns hat etwa 2 bis $2 \%^{\prime \prime \prime}$ Durchmesser. Sie ist im Stande bei günstigster Beleuchtung, d. h. wenn sie dem Lichte den Rücken kehrt, die vorgehaltenen Finger auf dunkelem Hintergrunde auf $8^{\prime}$ Entfernung zu zàhlen. Gegen das Licht oder im Freien erkennt sie nur Bewegungen der Hand auf 2\% Entfernung, Finger kann sie nicht zähIen. Sie wurde beiderseits durch Iridodesis am 27. August operirt. Bei ihrer Entlassung aus der Anstalt am 11. September erkennt sie nicht nur die kleinsten Objecte sehr genau, sondern zählt (und erkennt) im Freien bei sehr heller Beleuehtung die ausgestreckten Finger auf $20^{\circ}$ Entfernung. Dieselben Besultate ergaben die anderen (durch Iridodesis) operirten Fälle. Bei zwei Fällen von doppelseitigem Schichtstaar wurde auf dem einen Auge Iridodesis, auf dem anderen Iridectomie gemacht, da beide vorher mit Mühe grosse Druckschriften Lesen gelernt hatten, so konnte auch der gegenseitige Werth beider Operationsmethoden in Bezug auf das Sehen in den Nähe constatirt werden. Bei dem einen, P. K., hatte das Sehvermögen nach der Jäger'schsn Druckschriftenscala gemessen an dem durch Iridectomie operirten Auge nur um 8 Nummern zugenommen, während das durch Iridodesis operirte Auge um 12 Nummern sich verbessert hatte. Bei dem zweiten Th. Sch. hatte das Sehvermögen an dem durch Iridectomie operirten Auge um 4 Nummern, dagegen an dem durch Iridodesis operirten um 6 Nummern zugenommen.

4. Bei einem vierten Fall. Ch. D. aus Hamburg, 33 Jahre alt, bestand nur am rechten Auge Schichtstaar, das andere Auge hatte ein normales Sehvermögen. Die getrübte Schichte der Linse erstreckte sich (im Centrum 
stark saturirt, nach der Peripherie hin weniger intensiv) bis zu einer halben Linie vom Rande der Linse. Das Sehvermögen beschränkte sich bei normaler Pupillenweite auf das Erkennen der Bewegung einer Hand bei dunkelm Hintergrunde auf $1 / 2^{\prime}$ Entfernung, also ein so niederer Grad, wie man ihn nur bei einer ganzen Alterscataract finden kann. Ausserdem bestand ein Strabismus divergens von $1 \frac{1}{2}$ Linien. Die Iridodesis wurde am 17. Juni vorgenommen und am 1. Juli, am Tage der Entlassung aus der Anstalt war sie im Stande, die feinste Druckschrift (Jäger Nr. 3.) fliessend zu lesen. Gleichzeitig war durch Ermöglichung des Sehens mit beiden Augen der Strabismus divergens verschwunden.

5. Herr St., 28 Jahre alt, aus Nordamerika, hat in seinem 15. Lebensjahre einen Typhus überstanden und bemerkte alsbald nachher eine Abnahme seines bis dahin angeblich vollkommen normalen Sehvermögens. Beide Augen trübten sich so rasch, dass in seinem 16. Jahre eine "Staaroperation" an seinem linken Auge vorgenommen werden musste. Dieselbe bestand entweder in der Discision oder Reclination des Staars. 1/2 Jahr nach der Operation erlangte Patient an diesem Auge ein gutes Sehvermögen; er konnte mit entsprechenden Convexgläsern in die Nähe und in die Entfermung gut sehen. Das Sehvermögen erhielt sich, ohne dass irgend eine auffallende Affection eingetreten war, gut bis vor 2 Jahren, wo dasselbe ohne nachweisbare Ursache, d. h. ohne subjective Beschwerden innerhalb weniger Stunden schnell abnahm und bis jetzt in demselben Zustande verblieb.

Die Untersuchung des linken Auges ergibt eine erhebliche Gesichtsfeldbeschränkung; es fehlt der innere obere Quadrant und die Hälfte des äusseren. Der Kranke kennt mit Mühe die vorgehaltenen Finger auf 2 Fuss Entfernung. Bei erweiterter Pupille bemerkt man zahlreiche Kapselreste, welche noch Linsensubstanz in sich 
schliessen, und mit Hülfe des Augenspiegels eine Parthie der abgelösten Netzhaut nach aussen und unten im Glaskörper flottiren. Die Netzhautablösung ist wahrscheinlich den im Glaskörper befindlichen, der allmähligen Schrumpfung verfallenden Exsudatsträngen zuzuschreiben. Das rechte Auge zeigt einen Schichtstaar von scharf begrenzter Trübung mit ziemlich breiter ungetrübter Corticalis. Pat. lies't mit diesem Auge Jäger Nr. 14 mit einer stenopäischen Brille Nr. 13. Er erkennt und zählt, dem einfallenden Lichte den Rücken kehrend, Finger auf 6 Fuss Entfernung, gegen das Licht auf 21/2 Fuss.

Den $5 / \mathrm{II}$, wurde eine Iridodesis nach unten und innen gemacht.

Den 6/II. die Ligatur wird abgetragen, da sie nur noch an einem schmalen Streifchen Iris hängt.

Den 8/II. Verband bleibt weg. Die Wunde vernarbt.

Den 26/II. Pat. wird getheilt entlassen. Er sieht vortrefflich in die Entfernung. Er erkennt einzelne Bäume auf einem $1 / 2$ Stunde entfernten Berge, erzählt und erkennt im Freien ohne Kopfbedeckung Finger auf 30 Fuss Entfernung, ohne die mindesten Blendungserscheinungen und und lies't bequem Nr. 2. der Jäger'schen Schriftproben, und freut sich namentlich darüber, dass er des Tragens der lästigen Staarbrille überhoben ist.

6. Carl R., 8 Jahr alt, von Bad Ems, ein schwächlich gebauter Knabe, der in seinen ersten Lebensjahren an hydrocephalischen Erscheinungen gelitten, jetzt noch mit chronischer Otorrhöe behaftet, ist in seiner geistigen Entwickelung sehr zurückgeblieben, vielleicht auf Grund des ihm in hohem Grade mangelnden Gesichtssinnes. Er leidet sehr stark an Blendungserscheinungen und hat deshalb eine stark nach vorn gebeugte Haltung des Kopfes angenommen. Die Untersuchung der Augen ergibt beiderseits Schichtstaar von gleicher Grösse; der scheinbare Durchmesser der getrübten Linsenschichte beträgt 
5 Millim., die Breite der Iris bei ad maximum dilatirter Pupille 2 Millimeter. -

Der vorderen Kapsel liegen beiderseits nach innen punktförmige Trübungen auf, welche links zu einer sternförmigen Figur gruppirt sind. Die getrübte Linsenschichte sendet von ihrem Aequator kurze radiäre Ausläufer in die ungetrübte Corticalis aus. Ueber den Grad des Sehvermöpens konnte kein bestimmtes Urtheil gewonnen werden wegen der geistigen Unfähigkeit des Knaben. Kleinere Gegenstände wurden mehr durch den Tastsinn erkannt.

Am 10/x. wurde eine Iridodesis nach innen gemacht.

Den $11 / \mathrm{x}$. keine Reaction. Den $12 / \mathrm{x}$. die Ligaturen haben beiderseits durchgeschnitten und liegen im Conjunctivalsack, keine Reaction, der innere Rand der getrübten Linsenschichte wird von dem äusseren Rande der ganz nach innen gelagerten Pupille, nicht vollständig verdeckt. Dieselbe reagirt sehr lebhaft auf Lichteinfall.

Am 27/x. geheilt entlassen. Auffallend war, welche günstige Veränderungen in dem Verhalten des kleinen Patienten in den letzten Tagen während seines Aufenthalts in der Anstalt eingetreten war. Er schien um einige Jahre älter geworden, in Bezug auf die Aeusserungen seiner Geisteskräfte. Die Blendungserscheinungen waren gänzlich verschwunden, und es wurden kleine Gegenstände auf grössere Entfernungen, selbst gegen das einfallende Licht gehalten von dem Kranken erkannt. Wie grosse Mühe hätte man mit diesem Kranken gehabt, wenn man die Catarart durch Extraction hätte entfernen wollen, wie lange Zeit hätte die Nachbehandlung erfordert, wenn eine Discisio lentis vorgenommen worden wäre, abgerechnet die Unzuträglichkeit des Tragens einer Staarbrille bei einem Kinde, das Lesen lernen soll Eine Iridectomie würde wohl auch eben so rasch (der Kranke wurde am 17. Tage nach der Operation entlassen) 
zum Ziele geführt, aber gewiss nicht in dem Grade die Blendungserscheinungen beseitigt haben. -

Den 4/IV./61. Heute wurde der kleine Kranke zum erstenmal wieder vorgestellt. Körperliche und geistige Entwickelung hatten ungemein günstige Fortschritte gemacht. Der Kranke hatte während des letzten Monats zum erstenmal Unterricht im Lesen und Schreiben erhalten. Eine nochmals vorgenommene Prüfung des Sehvermögens ergibt, dass das Erkennen von Personen auf 3 bis 400 Schritte möglich ist. Die heute vorgenommene Messung der Pupille ergab rechts bei stärkster Contraction, scheinbare Länge 3 Millimeter, Breite 1 Millimeter, bei starker Erweiterung 4 bis 5 Millimeter lang und 2 bis $2 \frac{1}{2}$ breit. Betrachten wir nun eine solche Verlagerung der Pupille bei Schichtstaar, gegenüber einer durch Iridectomie bewirkten Pupille, so erhalten wir folgende Resultate: Eine Pupille durch Iridectomie bei Schichtstaar angelegt, vermindert nicht das Quantum des diffusen Lichtes; im Gegentheil dasselbe wird noch vermehrt, es werden zwar durch die Vergrösserung der Pupille auch rein gebrochene Strahlen der Retina zugeführt, allein die Blendung besteht fort, da weitaus der grösste Theil der Pupille der getrübten Linsenschichte gegenüber liegt.

Solche Operirte sehen im Freien oder bei reflectirtem Lichte, bei Sonnenschein nicht viel besser, als vor der Operation. Sie sind immer bemüht, durch ihre Stellung, durch Beugen des Kopfes nach vornen, ihre Lichtscheu zu vermindern. Das Sehen in grössere Entfernung ist ihnen unmöglich, mindestens sehr beschwerlich, die Verlagerung der Pupille gestattet dagegen solchen Kranken

1) ein sehr präcises Sehen in der Nähe,

2) es findet keine Lichtscheu statt, die Kranken können bei jeder Beleuchtung gleich gut sehen, weil sich der Sphincter pupillae in seiner unge- 
gestörten Thätigkeit immer der Quantität

des einfallenden Lichtes adaptirt, und

3) können sie auf eine weit grössere Entfernung deutlich sehen. -

Die Frage, ob nicht der Schichtstaar durch eine Staaroperation $\mathrm{zu}$ beseitigen sei, fällt also nach diesen Erörterungen ganz weg, da kein Operateur eine Staaroperation in dem Falle unternehmen wird, wo er durch eine ungefährliche Operation dieselben, ja noch grössere Vordertheile für das Sehvermögen erlangen kann.

Aus den mitgetheilten Krankengeschichten glaube ich den Schluss ziehen zu dürfen, dass die Verlagerung der Pupille durch die Iridodesis als das vorzüglichste Operationsverfahren bei Schichtstaar aufzustellen ist, namentlich den verschiedenen Arten von Staaroperationen, so wie auch der Iridectomie gegenüber. Es ist selbstverständlich, dass diejenigen Fälle von Schichtstaar sich nicht zu dieser Methode eignen, bei welchen die getrübte Linsenschichte beinahe bis zur Peripherie der Linse geht. Es ergibt sich aber, wie aus den ferner folgenden Krankengeschichten $\mathrm{zu}$ ersehen ist, eine weitere Indication für Operationsmethode, die selbst jede Concurrenz der Iridectomie ausschliesst. Ich meine hiermit diejenigen Fälle von Sehstörungen, die durch Vorhandensein von Astigmatismus, mag derselbe von der Cornea oder der Linse ausgehen, bedingt ist. Donders hat schon im 1. Heft des 7. Bandes des Archiv's darauf hingewiesen, welche Vortheile die Verlagerung der Pupille hinter eine richtig brechende Parthie der Cornea in sich schliesst, wenn die der normalen Pupille entsprechenden Medien Astigmatismus hervorrufen. Eine einfache Iridectomie (ohne Einklemmung der Iris) kann diesen Zweck nicht erreichen, da sie die Quelle der anomalen Lichtbrechung nicht verstopft, während man durch das Herüberziehen des Pupillarrandes diesseits des pathologisch gewölbten 
Theils der Cornea auch deren Folgen umgeht. Am meisten ausgeprägt ist dieser Umstand bei Keratoconus. Es eignen sich jedoch auch viele Fälle von Hornhautflecken zu dieser Methode. Nach Donders ist fast bei jeder normalen Cornea ein höherer oder niederer Grad von Astigmatismus vorhanden, ganz besonders aber finden wir, selbst ohne Ophthalmeter und Ophthalmoskop zu constatiren, häufig diesen Zustand entwickelt bei den Folgezuständen von chronischen, central gelegenen Hornhautulcerationen. Die Hornhaut hat dann oft in der Nähe von Narben selbst ohne dass vordere Synechien vorhanden sind, ein hügeliges Ansehen durch die partiellen Hervortreibungen. Bei solchen Fällen wählt man sich die günstigste, normal brechende oder doch dem normalen Brechzustande am nächsten functionirende Parthie der Cornea, die ja fast immer mehr oder weniger peripherisch liegt, aus, um hinter ihr die neue Pupille zu placiren. Ich habe in einer grossen Reihe von Fällen ein sehr präcises Sehvermögen sowohl in der Nähe, als auch in der Entfernung wiederherstellen können, was durch Auwendung der Iridectomie nicht möglich gewesen wäre.

C. Th., 27 Jahr alt, Schuhmacher, stellte sich vor wegen einer im Verlauf der letzten Jahre langsam zunehmenden Schwäche des Gesichts. Der Kranke gab an, dass er von jeher kurzsichtig gewesen, dass ihm aber das Arbeiten bei seiner Profession in der letzten Zeit immer schwieriger geworden sei, so wie, das Blendungserscheinungen bei heller Beleuchtuug ihn sehr belästigten. Die nähere Untersuchung seiner Augen ergab beiderseits Keratoconus in nicht unbeträchtlichem Grade. Die Cornea des linken Auges zeigt bei vollkommener Transparenz eine kegelförmige Erhebung von etwa 2-3 Linien, das Centrum des Kegels genau entsprechend dem Centrum der Cornea. Mit diesem Auge war der Kranke 
im Stande noch Nr. 17. der Jäger'schen Schriftproben auf 16 bis 17" Entfernung zu lesen. Kleinere Buchstaben wurden nicht mehr erkannt, Concavgläser verbessern nicht das Selvermögen. -

Die Cornea des rechten Auges zeigte ebenfalls bei vollkommener Transparenz eine Erhebung von 1 bis 2 Linien, das Centrum des Kegels fiel etwas unter die Mitte der Cornea. Mit diesem Auge las der Kränke noch Nr. 3 der Jäger'schen Schrift auf 3 bis 4" Entfernung. - Da die hochgradige Gesichtsschwäche bei einer vollkommenen Klarheit der übrigen brechenden Medien und normalem Verhalten der Retina und Chorioidea, nur den von der Cornea ausgebenden Zerstreuungskreisen zugeschrieben werden konnte, so wurde am linken Auge als dem am weitesten vorgeschrittenen eine Iridodesis gemacht. Durch die am $7 / \mathrm{vI}$. vorgenommene Operation gelang es, die Pupille so peripherisch zu legen, dass der grösste Theil derselben dem noch ziemlich normal gewölbten Randtheil der Cornea gegenüber zu liegen kam. -

Bei der Entlassung des Kranken am 12/VI. war er im Stande Jäger Nr. 1 auf 2 " Entfernung zu lesen, die Blendungserscheinungen waren an diesem Auge gänzlich verschwunden. Die scheinbare Pupille betrug bei ihrer stärksten Contraction im Längendurchmesser 5 Millim., im Breitendurchmesser 21/3 Millimeter.

Ph. S., früher Militair, litt damals mehrere Jahre an granulöser Augenentzündung, die zu Ulcerationen mit consecutiver Narbenbildung auf der Cornea beider Augen Veranlassung gegeben hatte.

Das bessere rechte Auge zeigte mehrere isolirt durchscheinende Trübungen, die theils der Pupille gegenüber, theils im oberen Theil der Cornea liegen. Der Kranke lies't mit blossem Auge Jäger Nr. 3, stenopäisch Jäger 
Nr. 1. Im Freien ohne Kopfbedeckung zählt er Finger auf $35^{\prime}$ Enfernung.

Das linke Auge zeigt stärker getrübte Narbenmassen im Centrum der Cornea, die Pupille direct bedeckend, die ganze äussere Hälfte der Cornea hat einen leichten Anflug von Trübung, der innere untere Quadrant der der Cornea mit Ausnahme seiner, der Pupille entsprechenden Spitze frei von Trübung. Der Kranke lies't mit blossem Auge Jäger Nr. 14, stenopäisch Nr. 5 mühsam, zählt Finger auf $9^{\prime}$, im Freien oder gegen das Licht nur auf $8^{\prime}$ Entfernung. Den 30/I. Verlagerung der Pupille durch Iridodesis nach innen und unten. - Druckverband.

Den 31/1. Der Faden wird weggenommen, keine Reaction - Pflasterverband.

Den 1/II. Der Verband wird weggelassen, der Kranke lies't $\mathrm{Nr} .9$.

Den 4/II. Die Injection der Conjunctiva verschwunden. Pat. lies't Nr. 3.

Den 13/Ir. Entlassung des Kranken. Fr lies't Nr. 1 und zählt im Freien ohne Kopfbedeckung die Finger auf 40' Entfernung.

Es lassen sich ferner durch die Verlagerung der Pupille auch Sehstörungen beseitigen, die durch von der Linse ausgehenden Astigmatismus bedingt sind. Als Beispiel möge folgender Fall von Ectopia lentis congenita dienen. -

M. R., ein Knabe von 10 Jahren, wurde vom Vater vorgestellt wegen Kurzsichtigkeit des rechten und Schwachsichtigkeit des linken Auges, die angeblich von Geburt aus bestehen sollten. Die Untersuchung des rechten $\mathrm{Au}$ ges ergab einen Bulbus von normaler Grösse, normaler Consistenz, keinen auffallend myopischen Bau. Iris von grau-grünlicher Farbe, normal gegen Licht reagirend, bei Bewegungen des Auges indessen stark schlotternd. Sehvermögen: lies't Jäger $\mathrm{Nr}$. 1, von 2 bis 4 " für die 
Ferne corrigirt nicht vollständig concav 5. Das Ophthalmoskop zeigte vollkommene Klarheit der brechenden Medien, und Sclerectasia posterior in geringem Grade. Nach Erweiterung der Pupille durch Atropin zeigte sich, dass die Linse in ihrem äquateriellen Durchmesser etwas verkleinert war, indem der Rand derselben schon bei gerade nach vorn gerichteter Stellung des Auges kaum von dem allerdings schmalen Irissaum bedeckt wurde, ferner war auffallend, dass die obere Hälfte des Linsenrandes mehrere starke, zackige oder wellenförmige Begrenzungen hatte, in geringerem Grade war dies auch nach unten der Fall (Unvollkommene fötale Entwickelung des Linsensystems.)

Das linke Auge zeigte äusserlich dieselben Eigenschaften, wie das rechte Auge. Das Schlottern der Iris war besonders in der unteren Hälfte stark, der obere Theil der Pupille (etwa $3 / 5$ derselben) bietet einen mattbräunlichen Reflex und contrastirt sehr gegen die unteren ${ }^{2} / 3$, die in tiefschwarzer Farbe erscheinen durch einen scharf abgeschnittenen, nach unten convexen Saum. Mit dem Augenspiegel bemerkt man in der Pupille, die oberen $3 / 3$ derselben von den unteren $2 / 5$ getrennt, durch einen mit seiner Convexität nach unten gerichteten Bogen, der nach oben und etwas nach hinten abschattirt ist, wie ein Aequatorialsegment der nach oben und etwas nach hinten dislocirten Linse, die übrigens vollkommen $\mathrm{i}^{\mathrm{n}}$ ihrer Stellung fixirt ist. Demgemäss erhält man auch 2 ophthalmoskopische Bilder des Augenhintergrundes, das nicht die Linse passirende aus der unteren Hälfte der Pupille, aufrecht, das die Linse passirende aus dem oberen Theil der Pupille umgekehrt bei Anwendung eines Convexglases. Patient erkennt mit diesem Auge Buchstaben von Jäger Nr. 15 und zählt gegen das Licht die Finger auf 8 Fuss. - Während stets 2 Bilder auf der Retina entstehen müssen, von dem das eine Zerstreuungs- 
kreise daselbst bildet, unterdrückt jedoch Pat. das eine, wie das Nichtvorhandensein von Doppelbildern beweist. Eine speciellere Prüfung erlanbt leider die geistige Entwickelung des Knaben nicht.

Die vorhandene Myopie (Sclerectasia posterior ist nachzuweisen) durch Concavgläser $\mathrm{zu}$ corrigiren gelingt nicht, eben so wenig gelingt es deutliche Bilder durch Benutzung von starken Convexgläsern zu erzielen, die in der Absicht vorgehalten wurden, den Kranken mit Benutzung des untern von der Linse freien Pupillaraum sehen zu lassen.

Indicirt ist zur Verbesserung des Sehens der Ausschluss eines der Bilder, begreiflicher Weise der Ausschluss des ohne die Linse gebildeten, demgemäss eine Verlagerung der Pupille nach oben. -

Den $4 / \mathrm{x}$. Ausführung der Operation durch Iridodesis nach oben;

Den 5/x. Keine Reaction. Den 6/x. Die Ligatur hat durchgeschnitten, Verband wird weggelassen. Den 9/x. der Kranke lies't Nr. 9.

Den 10/x. Wahrscheinlich durch zu langes Untersuchen mit dem Augenspiegel trat eine leichte Reizung des Auges ein; es werden deshalb 4 Blutegel applicirt.

Den 24/x. Geheilt entlassen, lies't Jäger Nr. 5 und zählt Finger im Freien auf $20^{\prime}$ mit -10 auf $24^{\prime}$ Entfernung. Wahrscheinlich würde das Sehvermögen noch besser ausgefallen sein, wenn die Lage der Linse nicht eine schief nach hinten gerichtete gewesen wäre. 